\title{
A PACKAGE FOR IMPEDANCE/ADMITTANCE DATA ANALYSIS
}

\author{
Bernard A. BOUKAMP \\ Twente University of Technology, Department of Chemical Technology, Laboratory for Inorganic \\ Chemistry and Materials Science, P.0.Box 217, 7500 AE Enschede, The Netherlands.
}

An outline is given of a Basic computer program which facilitates the analysis of frequency dispersion data. With this program an equivalent circuit, and starting values for the corresponding circuit parameters, can be extracted from the dispersion data. A circuit description together with crude parameter values form an essential requirement for a subsequent NLLSF procedure. A brief description is given of a frequency dispersion simulation program, also written in Basic, which can be used to compare measured data with a calculated response. Both programs employ the Circuit Description Code (CDC), thus allowing the use of a variety of equivalent circuits. The use of both programs is demonstrated with the analysis of a dispersion measurement performed on a sample of Sn-doped $\mathrm{AgCrS}_{2}$, which is a pure ionic conductor.

\section{INTRODUCTION}

Impedance spectroscopy is frequentiy used in the study of electrochemical systems ${ }^{1-6}$. One of the advantages of this technique is that from the immittance plots of the dispersion data good visual information is obtained about the characteristics of the electrochemical system. The dispersion is generally analyzed with the use of an equivalent circuit as model, in which the various circuit elements are related to the respective processes in the system, e.g. ionic conductivity, double layer capacitance, Warburg diffusion, etc.. In many cases the circuit parameters may be extracted using simple graphical means. However, if the time constants of the respective subcircuits are relatively close together the frequency dispersion cannot be devided in distinct separate regions. This is also true if elements with a fractional power dependence on frequency are present, e.g. Warburg or Constant Phase Elements ( $\mathrm{CPE}^{7-9}$ ), because the modulus of such an element varies sub-linear with frequency, extending its influence over a large frequency range in the dispersion.

In these cases all circuit parameters must be adjusted simultaneousiy, in order to fit the equivalent circuit response to the measured dispersion. This can be accomplished through the use of a special Non-Linear Least Squares Fit (NLLSF) procedure ${ }^{10-12}$. In order to use such a NLLSF procedure effectively one must know the shape of the equivalent circuit and have a set of adequate starting values for the adjustable circuit parameters.

This information can be obtained by a stepwise analysis of the dispersion data in an immittance diagram. The most prominent parts are located first and the corresponding elements are removed from the circuit, substracting their dispersion from the overal frequency dispersion. The use of personal computers, which have moderate graphics capabilities, can be of great advantage in this analysis.

In this paper an outline is given of such an analysis program. In many cases this program will yield a suitable equivalent circuit, together with an adequate set of starting values. for a subsequent NLLSF procedure. The intermediate and final fit results may be compared with the measured data using a dispersion simulation program, which will be discussed briefly. Both 
programs use the Circuit Description Code ${ }^{12,13}$ (CDC), which allows the use of a variety of equivalent circuits with these programs without a need for rewriting the source code.

\section{OUTLINE OF CIRCUIT ANALYSIS PROGRAM}

Crude values for the adjustable parameters are obtained through simple "graphical" means. The interface response of an ionic conductor can often be modeled as a total ionic resistance (grain boundary + ionic resistance) in series with a CPE element, sometimes combined with a capacitance $^{14}$. In this partial dispersion, in the impedance representation, a suitable point is selected through which a tangential line is drawn. The intersection with the real axis gives the total resistance, while the fractional exponent, $n$, of the CPE is found from the slope. The dispersion relation for the CPE is given by?

$$
Y^{*}(\omega)=Y_{0} \cdot(j \omega)^{n}
$$

The factor $Y_{0}$ is found from the imaginary value of the selected data point, $Z_{i}^{\prime \prime}$ :

$$
Y_{0}=-\sin \frac{n \pi}{2} / Z_{i}^{\prime \prime} \cdot \omega_{i}^{n}
$$

An estimate for the capacitance can be found by substracting at the low frequency limit, $\omega_{7}$, the calculated CPE response from the imaginary part of the measurement, $Z_{1}^{\prime \prime}$ :

$$
c=1 / \omega_{1}\left(Z_{1}^{\prime \prime}-\omega_{1}^{-n} \cdot \sin \frac{n \pi}{2} / Y_{0}\right)
$$

A similar procedure is used for the analys is of the bulk response at the high frequency limit of the dispersion, but now in the admittance representation. Here a resistance in parallel with a CPE and/or capacitance is obtained.

When there is little interference with the dispersion of the adjacent subcircuit, these values can be used for a subsequent simple NLLS-fit by the program, see below. Otherwise, the parameters for the adjacent subcircuit must be obtained in order to perform a NLLS-fit for the combined subcircuits. To this purpose, the CPE (and capacitance) dispersion is removed from the total dispersion, thus exposing the dispersion of the adjacent subcircuit more clearly.

Generally the next subcircuit can be regarded as a parallel combination of a CPE and a resistance (e.g. grain boundary immittance) in series with another resistance. This results in a depressed semi-circle in the immittance diagrams. By choosing a set of three data points the program can fit a circle, and give values for the resistances, as well as the CPE parameters.

The fitting of a set of subcircuits is performed in the impedance representation to a selected part of the dispersion curve. An analitical search procedure, which has been described elsewhere ${ }^{12}$, is used. The error function fits the real and imaginary parts simultaneously, using one weight factor:

$$
S=\sum_{i} w_{i}\left[\left(Z_{i}^{\prime}-Z^{\prime}\left(w_{i}\right)\right)^{2}+\left(Z_{i}^{\prime \prime}-Z^{\prime \prime}\left(w_{i}\right)\right)^{2}\right]
$$

where $Z_{j}^{*}=Z_{i}^{\prime}+j Z_{i}^{\prime \prime}$ is the measured data set and $Z^{*}(\omega)$ represents the calculated fit response. The weight factors are inversely proportional to the square of the modulus of the measurements:

$$
w_{i}=1 /\left|z_{i}^{*}\right|^{2} \text {, }
$$

which insures that all data sets contribute equally to the error function ${ }^{15}$.

The calculation of the circuit response in the fit procedure is based on a Circuit Description Code $(C D C)^{12,13}$, which uniquely represents the equivalent circuit. The CDC, which is interpreted by the program, acts as a set of pointers to a corresponding set of subroutines which calculate the individual responses of the different types of circuit elements. The partial derivatives of the error function with respect to the adjustable parameters, are calculated in the same subroutines and at the same time. This provides for a very compact source code for the fit procedure.

\section{DISPERSION SIMULATION PROGRAM}

Through the use of the $C D C$ this program can 
calculate the frequency dispersion of different complex equivalent circuits, which may include various diffusion related dispersions 13,16 . The calculated dispersion can be compared to actual measurements in both immittance diagrams and in a Bode plot.

The quality of a NLLS-fit result is best seen in a"Fit Quality" plot (FQ-plot). In this plot the relative deviations of the real, $\Delta_{r e}$, and imaginary, $\Delta_{i m}$, parts are plotted against $\log (\omega)$, with:

$$
\Delta_{r e_{i}}=\frac{Z_{i}^{\prime}-Z^{\prime}\left(\omega_{i}\right)}{\left|Z_{i}^{*}\right|} \text { and } \Delta_{i m_{i}}=\frac{z_{i}^{\prime \prime}-Z^{\prime \prime}\left(\omega_{i}\right)}{\left|z_{i}^{*}\right|}
$$

For a good fit these deviations should be distributed randomly around the frequency axis.

\section{EXAMPLE OF DATA ANALYSIS}

The use of the data analys is program is demonstrated with a frequency dispersion measurement of a sample of $\mathrm{AgCrS}_{2}$ doped with $10 \% \mathrm{SnS}_{2}$. This layer compound is a pure ionic conductor ${ }^{17}$. The ac-conductivity was measured on a sample with ionically blocking gold electrodes, using a Solartron 1250 FRA. The data acquisition and correction was performed with an Apple II computer. The frequency range was $655 \mathrm{mHz}$ to 65.5 $\mathrm{kHz}$. The dispersion, measured at $298 \mathrm{~K}$, is given in the admittance plot of fig. 1. From

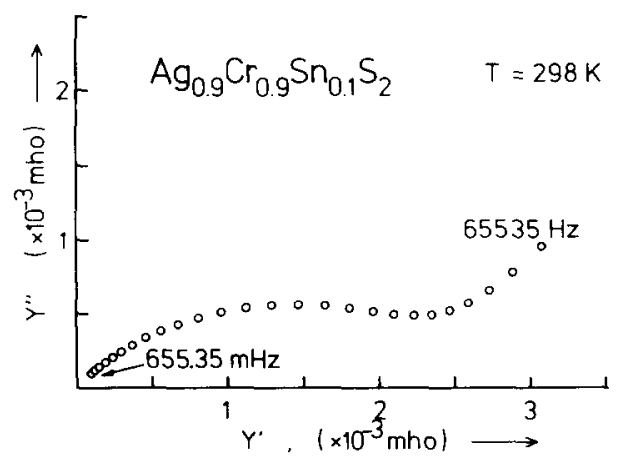

FIGURE 1

Admittance diagram of the measured dispersion.

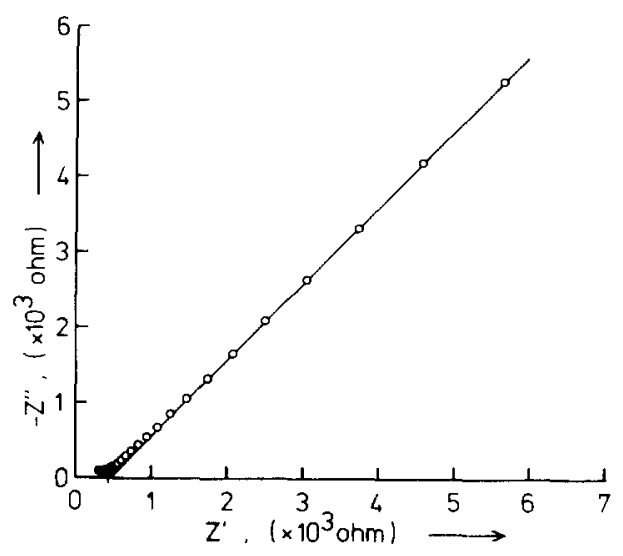

FIGURE 2

Impedance diagram showing the tangential line for the estimation of the interface parameters.

first observation the equivalent circuit seems quite simple, a bulk CPE in parallel with the ionic resistance, and an interface element (Warburg or CPE).

As the interface dispersion is most pronounced it is used for the first step in the analysis. With a selected point the tangential line option (eqs 2,3) is used, resulting in crude values for the ionic resistance and the CPE parameters, fig. 2. With the NLLS-fit an optimized set is obtained. Next the response of the interface CPE is substracted from the entire data set. The resulting dispersion is given in fig. 3. This admittance plot obvious1y represents two interfering subcircuits.

The parameters of the high frequency (bulk) CPE are estimated using the tangential line option in the admittance representation (fig. 3). A subsequent NLLS-fit is not useful as the influence of the medium frequency dispersion might extend into the high frequency dispersion. As an approximation the bulk CPE dispersion is substracted from the modified data set, leaving a depressed semi-circle. A circle is fitted by the program through three selected points in this dispersion, yielding values for the resis- 


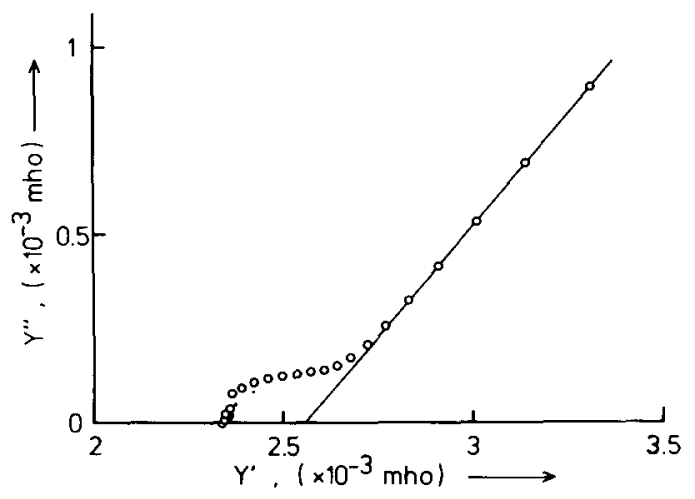

FIGURE 3

Admittance diagram of the modified data set showing the tangential line used in the estimation of the parameters of the bulk CPE.

tances and the corresponding (grain boundary) CPE.

These values, together with the parameters of the bulk CPE, are used in a final NLLS-fit of the modified data set in order to optimize the parameter set. The circuit used in this fit is based on the equivalent circuit of fig. 5, CDC: $(R P)(R P)$. The final result is given in table 1 .

The frequency dispersion, based on the final parameter set, is compared with the total measured dispersion in the FQ-plot of fig. 6, using the dispersion simulation program. From

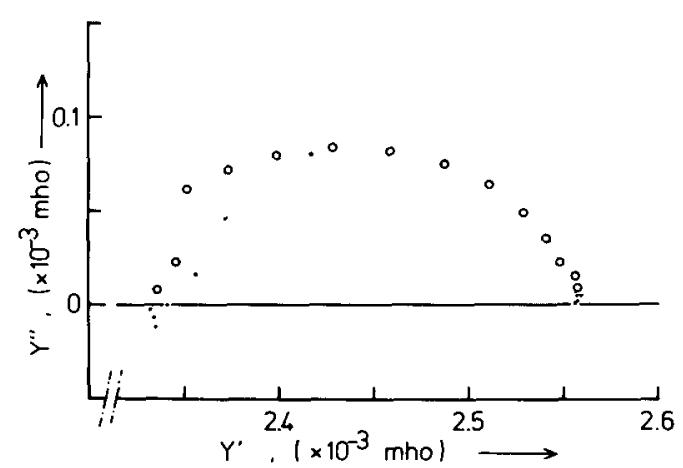

FIGURE 4

Admittance diagram of the resulting (grain boundary) dispersion.

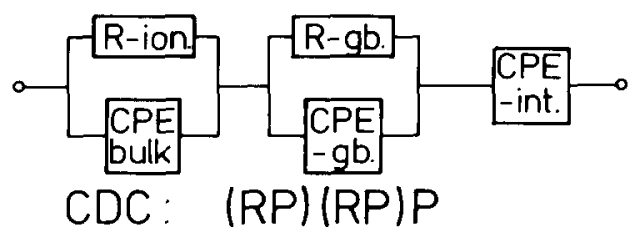

FIGURE 5

Schematic representation of the equivalent circuit used in the dispersion analysis.

this plot it can be seen that the extracted circuit and parameter set adequately describe the measured dispersion. In this case the general NLLSF procedure is only needed for obtaining a set of error estimates for the circuit parameters. The result of a complete fit performed with the program "EQIVCT" is given in the last column of table 1 .

\section{DISCUSSION}

No concise and formal procedure can be given for the analysis of frequency dispersion data. Each procedure strongly depends on the type of equivalent circuit assumed to represent the measured data best. The interactive dispersion analysis program, outlined in this paper, can be of help in many cases. Its use does require some experience in frequency dispersion analysis.

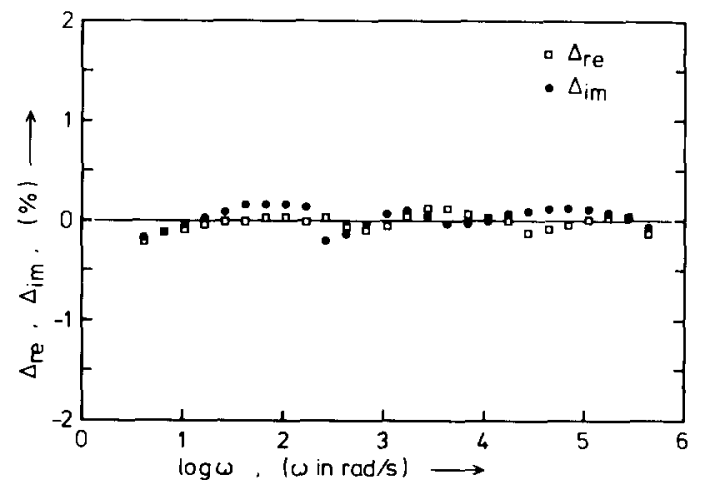

FIGURE 6

FQ-plot for the final parameter set obtained with the dispersion analysis program. 
TABLE 1

Parameter results obtained with the data analysis program and the general immittance fit program "EQIVCT"12. The final relative error estimates are also given.

\begin{tabular}{|c|c|c|c|c|}
\hline Element: & $\begin{array}{l}\text { Param- } \\
\text { eter: }\end{array}$ & $\begin{array}{c}\text { data analysis } \\
\text { program: }\end{array}$ & $\begin{array}{l}\text { NLLSF-prog. } \\
\text { "EQIVCT": }\end{array}$ & $\begin{array}{l}\text { rel. } \\
\text { err: }\end{array}$ \\
\hline R-ionic & $R$ & 391 & 389 & $0.3 \%$ \\
\hline CPE-bu $7 \mathrm{k}$ & $\left\{\begin{array}{l}Y_{0} \\
n\end{array}\right.$ & $\begin{array}{l}9.0 \times 10^{-7} \\
0.55\end{array}$ & $\begin{array}{l}8.2 \times 10^{-7} \\
0.56\end{array}$ & $\begin{array}{l}5 \% \\
0.7 \%\end{array}$ \\
\hline$R-g b$. & $R$ & 38 & 62 & \\
\hline CPE-gb. & $\left\{\begin{array}{l}y_{0} \\
n\end{array}\right.$ & $7.1 \times 10^{-5}$ & $1.5 \times 10^{-4}$ & $18 \%$ \\
\hline CPE-int. & $\left\{\begin{array}{l}y_{0} \\
n\end{array}\right.$ & $6.66 \times 10^{-5}$ & $6.64 \times 10^{-5}$ & $0.1 \%$ \\
\hline
\end{tabular}

The NLLS-fit option is an useful tool for obtaining simultaneously the parameters of two interfering subcircuits. Its use requires, however, that the data has been corrected previously for instrumental distortions. Also special care must be taken as the precission and range of the floating point numbers is rather limited in the present generation of personal computers. To prevent overflow errors, the data must be multiplied with a constant scaling factor, $f_{S C}$ :

$$
f_{s c}=1 / \sqrt{\left|z_{1}^{*}\right| \cdot\left|z_{h}^{*}\right|}
$$

where $\left|Z_{j}^{\star}\right|$ and $\left|Z_{h}^{*}\right|$ are the vector length of the impedances at the lowest and highest measured frequencies, $\omega_{1}$ and $\omega_{h}$. This procedure centers the values of the dispersion measurements around 1. The obtained parameters must be re-scaled accordingly.

It is often possible that, within the error 7 imits, the same data set may be interpreted with more than one equivalent circuit. The example presented here can be analysed with the bulk-CPE parallel to $R_{j}$ and the grain boundary dispersion (CDC: ' $\left.(P(R(R P))) P^{\prime}\right)$ but also with a Randless type circuit for the interface, without grain boundary, (CDC: '(RP) $\left.(P(R P))^{\prime}\right)$. Measurements at different temperatures are needed in order to elucidate the correct equivalent circuit.
The disadvantage of Basic programs is the comparatively slow execution. This may be improved by translating the programs in FORTRAN or Pascal. A7so the new generation of 16-bit personal computers will greatly improve the execution time of these programs.

The source code of the programs discussed in this paper may be obtained from the author.

\section{REFERENCES}

1. P.H. Bottelberghs, Low-frequency measurements on solid electrolytes and their interpretations, in: Solid Electrolytes, eds. P. Hagenmuller and W. van Gool (Academic Press, New York, 1978) pp. 145-172.

2. J.R. Macdonald and J.A. Garber, J. Electrochem. Soc. 124 (1977) 1022.

3. I.D. Raistrick and R.A. Huggins, in: Proc. Symposium and Workshop on Advanced Battery Research and Design, Argonne National Laboratory 76-8 (1976) B277.

4. J.R. Macdonald, in: Electrode Processes in Solid State Ionics, eds. M. Kleitz and

J. Dupuy (D. Reide 1, Dordrecht, 1976) p. 149 .

5. D.R. Franceschetti, J. Schoonman and J.R. Macdonald, Solid State Ionics 5 (1981) 617.

6. B.A. Boukamp and G.A. Wiegers, Solid State Ionics 9/10 (1983) 1193.

7. P.H. Bottelberghs and G.H.J. Broers, J. Electroanal. Chem. 67 (1976) 155.

8. I.D. Raistrick, C. Ho and R.A. Huggins, J. Electrochem. Soc. 123 (1976) 1469.

9. J.R. Macdonald, Solid State Ionics 13 (1984) 147.

10.J.R. Macdonald, A. Hooper and A.P. Lehnen, Solid State Ionics 6 (1982) 65.

11.J.R. Macdonald, J. Schoonman and A.P. Lehnen, J. Electroanal. Chem. 131 (1982) 77.

12 B.A. Boukamp, submitted for publication.

13 B.A. Boukamp, Internal Report CT85/177/128, Twente University of Technology, 1985.

14.B.A. Boukamp and R.A. Huggins, Mat. Res. Bu11 13 (1978) 23.

15.P. Zoltowski, J. Electroana1. Chem. 178 (1984) 11.

16.B.A. Boukamp, Internal Report CT85/178/128, Twente University of Technology, 1985.

17.T. Hibma, D. Brüesch and S. Strässler, Solid State Ionics 5 (1981) 481. 